\title{
Les légitimations complexes de l'internationalisation de l'enseignement supérieur : le cas de la mobilité des étudiants maghrébins en France et au Québec Complex legitimations in the internationalised higher education system : the mobility of students from the Maghreb to France and to Quebec
}

\author{
Stéphanie Garneau et Caroline Bouchard
}

Volume 42, numéro 2, automne 2013

Transformations des mobilités étudiantes sud-nord : approches démographiques et sociologiques

URI : https://id.erudit.org/iderudit/1020608ar

DOI : https://doi.org/10.7202/1020608ar

Aller au sommaire du numéro

Éditeur(s)

Association des démographes du Québec

ISSN

0380-1721 (imprimé)

1705-1495 (numérique)

Découvrir la revue

Citer cet article

Garneau, S. \& Bouchard, C. (2013). Les légitimations complexes de l'internationalisation de l'enseignement supérieur : le cas de la mobilité des étudiants maghrébins en France et au Québec. Cahiers québécois de démographie, 42(2), 201-239. https://doi.org/10.7202/1020608ar

\section{Résumé de l'article}

Cet article offre une analyse, dans une perspective comparative France-Québec, des différentes modalités d'adhésion au processus d'internationalisation de l'enseignement supérieur. Il traite plus spécifiquement de l'une des dimensions de l'internationalisation des universités, les mobilités d'étudiants étrangers, en s'intéressant à des flux en importance dans ces deux sociétés d'accueil : ceux en provenance du Maroc, d'Algérie et de Tunisie. La démonstration prend appui sur des entretiens réalisés auprès de trente acteurs institutionnels, sur des données statistiques ainsi que sur des documents publics. L'article montre que les acteurs rencontrés en France et au Québec se trouvent au centre de deux tensions principales : la première, entre la logique marchande et la mission culturelle et linguistique des universités ; et la seconde, entre un objectif d'accroissement de l'accueil d'étudiants étrangers et un impératif de gestion du risque migratoire. Il présente en quoi chacune de ces tensions appelle, pour susciter leur réconciliation et l'adhésion des acteurs, des procédés de légitimation complexes. L'article éclaire ces processus de légitimation à l'aide des flux d'étudiants maghrébins dans ces deux sociétés. Il conclut sur deux catégories normatives - le " bon immigrant " au Québec et le « bon étudiant étranger " en France - qui tendent à s'imposer à travers ces opérations de pensée. 
Cahiers québécois de démographie

Vol. 42, $\mathrm{n}^{\mathrm{o}}$ 2, automne 2013, p. 201-239

\title{
Les légitimations complexes de l'internationalisation de l'enseignement supérieur: le cas de la mobilité des étudiants maghrébins en France et au Québec
}

\author{
STÉPHANIE GARNEAU* ET CAROLINE BOUCHARD ${ }^{\star *}$
}

\begin{abstract}
Cet article offre une analyse, dans une perspective comparative France-Québec, des différentes modalités d'adhésion au processus d'internationalisation de l'enseignement supérieur. Il traite plus spécifiquement de l'une des dimensions de l'internationalisation des universités, les mobilités d'étudiants étrangers, en s'intéressant à des flux en importance dans ces deux sociétés d'accueil: ceux en provenance du Maroc, d'Algérie et de Tunisie. La démonstration prend appui sur des entretiens réalisés auprès de trente acteurs institutionnels, sur des données statistiques ainsi que sur des documents publics. L'article montre que les acteurs rencontrés en France et au Québec se trouvent au centre de deux tensions principales: la première, entre la logique marchande et la mission culturelle et linguistique des universités; et la seconde, entre un objectif d'accroissement de l'accueil d'étudiants étrangers et un impératif de gestion du risque migratoire. II présente en quoi chacune de ces tensions appelle, pour susciter leur réconciliation et l'adhésion des acteurs, des procédés de légitimation complexes. L'article éclaire ces processus de légitimation à l'aide des flux d'étudiants maghrébins dans ces deux sociétés. Il conclut sur deux catégories normatives - le «bon immigrant» au Québec et le «bon étudiant étranger» en France - qui tendent à s'imposer à travers ces opérations de pensée.
\end{abstract}

English abstract p. 239

\section{INTRODUCTION}

$\mathrm{L}$ e fait semble avéré : le secteur public universitaire constitue désormais un marché international «libéralisé » dont une partie des services éducatifs possède de plus en plus une vocation marchande (Agasisti et Catalano, 2006; Organisation de coopération et de développement économiques [OCDE], 2004). Il y a à peine vingt-cinq ans, pourtant, «l'ensei-

* Université d'Ottawa, Ottawa, Canada (stephanie.garneau@uottawa.ca)

**Université d'Ottawa, Ottawa, Canada (cbouco85@uottawa.ca) 
gnement supérieur transnational » (Vincent-Lancrin, 2008) — c'est-à-dire la mobilité internationale des étudiants et des enseignants-chercheurs et la délocalisation des programmes de formation et des établissements d'enseignement supérieur — répondait encore majoritairement à des intérêts culturels et d'aide au développement (Borgogno et Streiff-Fénart, 1997; Garneau, 2008; Simon, 2000). Bien qu'en partie géostratégiques, ces motivations n'appelaient pas d'instruments politiques particuliers de valorisation ni de recrutement.

Depuis la signature en 1995 de l'Accord général sur le commerce des services (AGCS) et l'inclusion corrélative des services éducatifs au sein de l'Organisation mondiale du commerce (OMC), et bien que ces enjeux culturels et d'aide au développement ne se soient pas complètement effacés du paysage, l'internationalisation de l'enseignement supérieur dépasse largement le cadre strict des politiques de l'enseignement supérieur pour embrasser des intérêts proprement économiques. La managérialisation grandissante des universités influe de plus en plus sur leur vocation d'enseignement et de recherche, assujettie aux besoins de l'industrie plutôt que tournée vers la réflexion critique et les finalités poursuivies par la société ${ }^{1}$ ainsi que sur leur gestion, calquée sur le modèle de l'entreprise privée (le «new public management»). Ce mouvement global s'accompagne de la rhétorique de l'économie du savoir (Castells, 2001; Milot, 2003; Godin, 2006) et fait des universités des organisations stratégiques et concurrentielles pour les décideurs politiques (Bruno, 2008; Musselin, 2008).

Le processus d'internationalisation des universités avance donc main dans la main avec celui de leur managérialisation — bien que ni entièrement ni exclusivement —, cela dans la très grande majorité des systèmes nationaux d'enseignement supérieur. Des organisations comme l'OCDE et l'Organisation des Nations Unies pour l'éducation, la science et la culture (UNESCO) contribuent d'ailleurs, sur la base d'indicateurs le plus souvent mesurés quantitativement, à nourrir ce constat d'une convergence des orientations de l'enseignement supérieur à l'échelle internationale ${ }^{2}$. Elles tendent ainsi à faire oublier que l'implantation d'un processus international passe néanmoins par des acteurs concrets, qui ont intérêt à l'actua-

1. Pour une critique du tournant économiciste des universités, voir entre autres Freitag (1995), Giroux (2006) et le numéro 33 du M.A.U.S.S. (2009).

2. À l'instar de ce qu'a observé Hassentefeul (2005) à propos de la tendance à la libéralisation des systèmes d'assurance maladie en Europe. 
liser ${ }^{3}$. Aussi peut-on supposer que l'intensité de ce grand processus global qu'est l'internationalisation de l'enseignement supérieur dans un but de plus en plus marchand, sa traduction effective en mesures et dispositifs locaux et ses modalités de légitimation politique diffèrent selon les contextes sociétaux. La question devient alors moins de prouver qu'il y a bien des politiques publiques de l'enseignement supérieur qui vont dans le sens d'une internationalisation et d'une managérialisation dans la plupart des contextes sociétaux que de comprendre comment on passe concrètement de la tendance globale à son actualisation localisée. Moins qu'à un consensus et à un sens partagé, l'internationalisation et la managéralisation invitent plutôt à explorer les enjeux et les intérêts parfois contradictoires, entre organisations mais aussi chez un même acteur, qui concourent à leur coloration singulière selon les différents contextes sociétaux. Notre intention ne sera donc pas ici d'étudier leurs effets sur les pratiques ou structures de la gouvernance universitaire dans une perspective séquencée et surplombante (top-down $)^{4}$, ni leurs conséquences sur les étudiants étrangers 5 . Nous chercherons plutôt à comprendre comment certains acteurs sociaux se sont approprié une « idée » performative a priori généralisée (l'internationalisation de l'enseignement supérieur à vocation marchande), en lui injectant du sens en fonction des réalités qui sont les leurs et des rapports de pouvoir dans lesquels ils sont impliqués.

Nous avons pour cela privilégié une focale microscopique (Glaser et Strauss, 2012; Revel, 1996), c'est-à-dire que nous avons favorisé l'expérience et le discours d'acteurs concrets pour appréhender la complexité des situations d'adhésion à une mouvance à première vue globale et standardisante. Nous verrons que c'est cette réappropriation symbolique plurielle qui permet à cette mouvance, observable dans la plupart des pays, de "prendre vie » localement, dans une perspective non pas de nécessité mais de processus sociaux situés. Nous constaterons que si des catégories normatives tendent à émerger des discours et à s'imposer par-delà les tensions observées, certaines pratiques de résistance sont néanmoins perceptibles.

3. Selon Hassentefeul (2005: 127), « [1]'objectivation statistique donne une légitimé scientifique aux acteurs visant à diffuser [une] orientation » spécifique, en conférant un caractère de nécessité à la convergence internationale. La mouvance transnationale devient ainsi «partie intégrante de l'argumentation ». Elle devient une évidence, un « cela va de soi».

4. Comme tendent à le faire certaines analyses « classiques» des politiques publiques et des réformes en matière d'éducation (Abdous, 2011; Legrand, 1988).

5. L'étude des parcours d'étudiants étrangers fait l'objet d'une seconde phase de recherche, en cours, portant sur les carrières de socialisation professionnelles de Marocains diplômés au Québec. 
La démonstration prend appui sur des entretiens semi-directifs réalisés en France et au Québec en 2010-2011 auprès de trente acteurs institutionnels de l'enseignement supérieur, sur des données statistiques ainsi que sur des rapports publics, documents techniques et sites Internet. Dans ce souci d'ancrer les processus de légitimation et les catégorisations normatives qu'elles produisent dans des réalités concrètes, nous avons focalisé notre attention sur l'une des dimensions de l'internationalisation de l'enseignement supérieur, celle de la mobilité étudiante, en nous penchant sur les flux en provenance de trois pays spécifiques : le Maroc, l’Algérie et la Tunisie.

\section{LA MOBILITÉ DES ÉTUDIANTS MAROCAINS, ALGÉRIENS ET TUNISIENS EN FRANCE ET AU QUÉBEC}

Les établissements d'enseignement supérieur français et québécois font partie, à des degrés variables, des choix privilégiés des étudiants marocains, algériens et tunisiens. En effet, la France constitue de loin le premier pays de destination des étudiants originaires de ces trois pays. Selon l'UNESCO, elle accueillait en $200865 \%$ de tous les étudiants marocains en mobilité internationale, $86 \%$ de tous les étudiants algériens à l'étranger ainsi que $60 \%$ de tous les étudiants tunisiens dans cette situation (tableau 1). Les données comparables pour le Québec ne sont pas disponibles puisque l'UNESCO récolte des statistiques par pays et non par province. À titre indicatif, néanmoins, le Canada était à la même période le quatrième principal pays d'accueil des étudiants marocains à l'étranger, le deuxième principal pays d'accueil des étudiants algériens en mobilité internationale ainsi que la sixième principale destination des Tunisiens aux études à l'étranger.

TABLEAU 1 Les six principaux pays d'accueil des étudiants marocains, algériens et tunisiens en 2008

\begin{tabular}{|l|l|l|l|}
\hline & Maroc & Algérie & Tunisie \\
\hline 1 & France (26 998) & France (18 780) & France (10 812) \\
\hline 2 & Allemagne (3 699) & Canada (796)* & Allemagne (2 491) \\
\hline 3 & Espagne (2 735) & Royaume-Uni (335) & Roumaine (868) \\
\hline 4 & Canada (1 587)* & États-Unis (179) & Ukraine (611) \\
\hline 5 & Italie (1 207) & Allemagne (158) & Italie (611) \\
\hline 6 & États-Unis (1 133) & Suisse (127) & Canada (583)* \\
\hline
\end{tabular}

Source: UNESCO, cité dans Agence CampusFrance, $2011 \mathrm{f}$.

* Données de 2007 


\section{Les limites de la comparaison internationale des flux d'étudiants étrangers}

La comparaison des flux d'étudiants maghrébins vers la France et le Québec comporte certaines limites qui tiennent à la définition même de l'étudiant international dans chacune de ces sociétés. En ce qui concerne la France, les données rapportées ici ont été majoritairement puisées dans les "fiches pays » de l'Agence CampusFrance, qui dépend du ministère de l'Enseignement supérieur et de la Recherche (MESR). Sont comptées les inscriptions des étudiants de nationalité étrangère en France métropolitaine et dans les départements d'outre-mer (hors collectivités d'outre-mer) inscrits dans les universités pour une formation d'une durée d'au moins un an. Étant donné les difficultés d'obtention de la nationalité française (délais minimaux et lois diverses sur les conditions d'obtention de la nationalité), on comprend que les données de la France sur l'accueil des étudiants internationaux peuvent être grossies (Slama, 1999: 13) par rapport à d'autres sociétés qui octroient la nationalité plus rapidement, comme c'est le cas du Canada.

En effet, au Canada et au Québec, ni les résidents permanents ni les citoyens canadiens ne sont comptés parmi les étudiants étrangers. Plus spécifiquement, le statut légal des étudiants étrangers considérés par le ministère de l'Éducation, du Loisir et du Sport (MELS), d'où proviennent les données présentées dans cet article, est que ces derniers doivent être inscrits dans un établissement d'enseignement supérieur sans être ni citoyens canadiens, ni Indiens (selon la Loi sur les Indiens), ni résidents permanents. Dès lors qu'ils obtiennent la résidence permanente, ces étudiants sortent des statistiques sur les étudiants étrangers. Les effectifs réels d'étudiants internationaux accueillis dans les établissements d'enseignement supérieur québécois seraient donc supérieurs aux données officielles.

Ces données peuvent être mises en perspective grâce à un bref rappel de l'évolution récente des stratégies en matière d'internationalisation de l'enseignement supérieur et d'accueil d'étudiants étrangers adoptées en France et au Québec.

\section{L'accueil d'étudiants étrangers en France}

En France, une logique du soupçon a majoritairement prévalu entre la fin des années 1970 et le début des années 2000. Plusieurs mesures restrictives 
ont eu pour but d'empêcher l'entrée des "faux étudiants » (Borgogno et Streiff-Fénart, 1997; Slama, 1999). Entre 1990 et 1998, le nombre d'étudiants étrangers en France est passé de 161148 à 149295 (Conseil national pour le développement de la mobilité internationale des étudiants [CNDMIE], 2004: 10). La baisse a été encore plus marquée pour les étudiants du Maghreb puisqu'ils étaient 48000 inscrits dans les universités françaises en 1990 contre seulement 35000 en 1998 (Coulon et Paivandi, 2003:14).

À partir de la fin des années 1990 se dessine un changement des intentions politiques françaises, à la faveur, tout comme dans le monde anglosaxon, d'une offensive en matière d'internationalisation de l'enseignement supérieur. En 1998, l'Agence ÉduFrance est créée en vue de promouvoir l'offre éducative française à l'étranger et d'améliorer les conditions d'accueil des étudiants étrangers en France. Le nombre d'étudiants internationaux remonte alors à 221567 en 2002, soit un peu plus de 72000 inscrits qu'en 1998 (CNDMIE, 2004:10). Mais la loi pour la sécurité intérieure de 2003 (Loi Sarkozy II), qui abroge notamment l'obligation de motiver les refus de visa, s'accompagne d'une nouvelle diminution de la délivrance de visas de long séjour. Selon les données de l'UNESCO, en effet, les effectifs d'étudiants maghrébins (incluant la Libye) en France passent alors de 64800 en 2004 à 54690 en 2008 (Agence CampusFrance, 2011f).

C'est dans ce contexte que l'Agence ÉduFrance devient en 2007 l'Agence CampusFrance et qu'est officialisée la Convention-cadre sur les Centres pour les études en France $(\mathrm{CEF})^{6}$. L'Agence CampusFrance est un opérateur ayant pour principale mission la promotion de l'enseignement supérieur français. À l'heure actuelle, on retrouve 145 espaces CampusFrance dans autant de postes diplomatiques français à l'étranger (CampusFrance, site Internet, 2013). La procédure CEF, quant à elle, est un « dispositif qui permet au candidat à des études en France de bénéficier d'un appui et de conseils pour l'ensemble de ses démarches jusqu'à la demande de visa et de suivre l'évolution de son dossier électronique " (CampusFrance, site Internet, 2013). Aujourd'hui, la procédure CEF est obligatoire dans 31 pays, dont l'Algérie, la Tunisie et le Maroc. Entre 2007 et 2010, le nombre d'étudiants originaires de ces trois pays croît de plus

6. La Convention-cadre sur les CEF a été établie à l'initiative du ministère des Affaires étrangères, du ministère de l'Éducation nationale, de l'Enseignement supérieur et de la Recherche, du ministère de la Culture et de la Communication, de la Conférence des présidents d'université, de la Conférence des directeurs des écoles françaises d'ingénieur et de la Conférence des grandes écoles. 
belle, passant de 50881 à 53 0637. En 2009, les Marocains, les Algériens et les Tunisiens forment respectivement les premier $(10,9 \%)$, troisième $(7,7 \%)$ et quatrième ( $4,5 \%)$ plus gros contingents d'étudiants étrangers en France. Ils se disputent les premiers rangs avec les Chinois, lesquels occupent la deuxième place avec $9,5 \%$ de la part totale des étudiants étrangers (Agence CampusFrance, 2011e : 24).

Les demandes de changement de statut faites par les étudiants sont grandement contrôlées en France. La Loi 2006-911, par l'article 311-11, accorde aux étudiants étrangers ayant obtenu un diplôme au moins équivalent au master sur le territoire français la possibilité d'obtenir une autorisation provisoire de séjour (APS) d'au plus six mois non renouvelable. L'objectif visé est de permettre l'acquisition d'une première expérience professionnelle en vue d'un retour dans le pays d'origine. L'octroi des APS de même que les changements de statut d'immigration ont cependant été compliqués par l'adoption de la controversée " circulaire Guéant» le 31 mai 2011, laquelle visait à diminuer l'immigration « en adoptant une approche qualitative et sélective $»^{8}$. Face aux tollés de protestations dans les milieux universitaires et de l'entreprise, le ministre de l'Intérieur présenta une nouvelle circulaire en janvier 2012, où il appelait à ce que l'examen des demandes de changement de statut et d'APS «ne se fasse pas au détriment de l'attractivité du système d'enseignement supérieur français, ni des besoins de nos entreprises en compétences spécifiques de haut niveau $»^{9}$. Si la circulaire elle-même a été abrogée le 31 mai 2012, avec l'arrivée au pouvoir du parti socialiste, le dispositif d'APS continue néanmoins à encourager le retour des étudiants dans leur pays.

\section{L'accueil des étudiants étrangers au Québec}

Du côté de la société québécoise, le gouvernement péquiste adopte en 2002 une «stratégie d'internationalisation de l'éducation québécoise » (Gouver-

7. Voir les Fiches pays de l'Agence CampusFrance pour l'Algérie (2011a), le Maroc (2011b) et la Tunisie (2011c). Cette augmentation ne permet toutefois pas d'apprécier les opérations de sélection resserrées qui ont tout de même cours avec la procédure CEF, lesquelles ont fait l'objet d'observations dans d'autres enquêtes (Spire, 2009). Nous y reviendrons dans l'analyse.

8. Voir http ://circulaire.legifrance.gouv.fr/pdf/2011/o6/cir_33321.pdf [consulté le 15 février 2013].

9. Voir http://combatsdroitshomme.blog.lemonde.fr/files/2012/o1/Circulaire_Accèsau-marché-du-travail-des-diplômés-étrangers-de-niveau-au-moins-équivalentau-Master-s.pdf [consulté le 19 février 2013]. 
nement du Québec, 2002). Cette stratégie a pour but d'assurer une meilleure coordination des initiatives d'internationalisation entreprises séparément par les différents acteurs de l'éducation au Québec. Elle s'inscrit sur fond de compétition internationale : le Québec veut éviter d'être marginalisé par le processus d'européanisation de l'enseignement supérieur, et plus spécifiquement de perdre l'intérêt de la France, son partenaire traditionnel.

En 2006, le ministère Affaires étrangères, Commerce et Développement Canada crée Édu-Canada, un service chargé de faire la promotion internationale de l'éducation au Canada. Deux ans plus tard, le gouvernement Harper procède au lancement d'une image de marque afin d'assurer la visibilité du Canada dans l'espace internationalisé de l'enseignement supérieur : "Imagine Education au/in Canada $»^{10}$. Le gouvernement fédéral entre alors de plain-pied dans l'internationalisation de l'enseignement supérieur.

Contrairement à la situation en France, où les étudiants internationaux paient les mêmes droits de scolarité que les étudiants nationaux, au Québec l'accueil d'étudiants étrangers constitue entre autres une stratégie de financement des universités - dans un contexte de désengagement de l'État - puisque ces derniers paient 7500 \$ de plus qu'un étudiant québécois, pour un total de plus de 9000 \$ par année. Cela étant dit, un nombre important d'étudiants est exempté de ces droits de scolarité supplémentaires en vertu d'ententes diverses (Conseil supérieur de l'économie [CSE], 2005 : 31). En 2002, 60,5\% des étudiants internationaux concernés par les droits supplémentaires en étaient exemptés. Les étudiants ayant majoritairement profité d'une entente entre les gouvernements en 2003 sont, dans l'ordre, les étudiants français, sénégalais et ivoiriens. Les étudiants tunisiens et marocains arrivent en septième et huitième positions des principaux bénéficiaires de l'exemption (CSE, 2005:34).

En 2010, la France est le principal pays de provenance des étudiants étrangers dans les universités francophones du Québec. Viennent ensuite le Maroc, la Tunisie, la Chine et l'Algérie. Le nombre d'étudiants en provenance de ces pays a évolué de manière fort différenciée entre 2001 et 2010. Parmi les plus importantes augmentations, la présence des Chinois a crû

10. Pour un aperçu du logo et de la vidéo promotionnelle, voir http ://www.educationau-incanada.ca/educationau-incanada/index.aspx ? lang=fra\&view $=\mathrm{d}$ [consulté le 15 février 2013]. 
de $182 \%$, celle des Français de 95,5\% et celle des Algériens, de $74 \%$ $(\text { tableau } 2)^{11}$.

TABLEAU 2 Étudiants étrangers inscrits dans le réseau universitaire québécois francophone selon le pays de citoyenneté et le trimestre d'automne

\begin{tabular}{|l|c|c|c|c|c|c|c|c|}
\hline Automne & Algérie & Maroc & Tunisie & Maghreb & Chine & France & Autres & Total \\
\hline $\mathbf{2 0 0 1}$ & 176 & 908 & 661 & 1745 & 146 & 3742 & 4829 & 10462 \\
\hline $\mathbf{2 0 0 2}$ & 152 & 898 & 785 & 1835 & 169 & 3990 & 5440 & 11434 \\
\hline $\mathbf{2 0 0 3}$ & 219 & 903 & 801 & 1923 & 214 & 4401 & 5719 & 12257 \\
\hline $\mathbf{2 0 0 4}$ & 166 & 865 & 658 & 1689 & 202 & 4663 & 5293 & 11847 \\
\hline $\mathbf{2 0 0 5}$ & 183 & 908 & 592 & 1683 & 226 & 4851 & 5274 & 12034 \\
\hline $\mathbf{2 0 0 6}$ & 183 & 967 & 532 & 1682 & 293 & 5451 & 5332 & 12758 \\
\hline $\mathbf{2 0 0 7}$ & 198 & 954 & 533 & 1685 & 347 & 5811 & 5175 & 13018 \\
\hline $\mathbf{2 0 0 8}$ & $\mathbf{2 6 2}$ & 958 & 619 & 1839 & 346 & 5794 & 5139 & 13118 \\
\hline $\mathbf{2 0 0 9}$ & 308 & 1016 & 704 & 2028 & 353 & 6570 & 5498 & 14449 \\
\hline $\mathbf{2 0 1 0 *}$ & 307 & 908 & 679 & 1894 & 412 & 7315 & 5769 & 15390 \\
\hline Variation & $\mathbf{7 4 , 4} \%$ & $\mathbf{0 , 0} \%$ & $\mathbf{2 , 7} \%$ & $\mathbf{8 , 5} \%$ & $\mathbf{1 8 2 , 2} \%$ & $\mathbf{9 5 , 5} \%$ & $\mathbf{1 9 , 5} \%$ & $\mathbf{4 7 , 1} \%$ \\
\hline Poids 2010 & $\mathbf{2 , 0} \%$ & $\mathbf{5 , 9} \%$ & $\mathbf{4 , 4} \%$ & $\mathbf{1 2 , 3} \%$ & $\mathbf{2 , 7} \%$ & $\mathbf{4 7 , 5} \%$ & $\mathbf{3 7 , 5} \%$ & $\mathbf{1 0 0 , 0} \%$ \\
\hline
\end{tabular}

Source: MELS (2011)

*données provisoires

Outre les exemptions de droits de scolarité supplémentaires, une autre mesure visant à favoriser l'accessibilité des systèmes d'enseignement supérieur canadiens pour les étudiants internationaux est adoptée en 2005 par le Gouvernement canadien. Les étudiants étrangers peuvent désormais occuper un emploi hors campus pendant leurs études. En 2008, le ministère de l'Immigration et des Communautés culturelles du Québec (MICC) officialise le rapprochement entre la migration pour études et l'immigration de travail en annonçant dans son Plan stratégique 2008-2012 son intention d'accueillir un plus grand nombre d'étudiants étrangers et d'accélérer les démarches d'obtention du statut de résident (MICC, $2008: 18$ ).

11. Notons que selon les données du MELS (2011), cette forte croissance des étudiants chinois s'observe également du côté des universités anglophones du Québec. Ils sont passés de 404 à 1211 durant la même période, une évolution de $200 \%$. La variation des étudiants maghrébins dans les universités anglophones est pour sa part plus modeste, soit de 3,3\% seulement (contre 12,3\% du côté des établissements francophones). 
En France comme au Québec, les étudiants originaires du Maroc, d'Algérie et de Tunisie constituent donc une part importante, sinon majeure, de l'ensemble des étudiants internationaux accueillis dans les établissements d'enseignement supérieur. Dans l'une et l'autre de ces deux sociétés d'accueil francophones, ils sont de plus en plus en concurrence avec les étudiants en provenance de Chine. En outre, les flux d'étudiants marocains, algériens et tunisiens sont affectés par certaines décisions des autorités publiques. Au-delà de ces orientations, dépeintes ici à grands traits, quelles sont les tensions à l'œuvre et quelles propriétés argumentatives sont mises de l'avant par les différents acteurs français et québécois pour justifier leur participation à l'internationalisation de l'enseignement supérieur - dans une perspective marchande - et à l'accueil d'étudiants en provenance du Maghreb? Que se dégage-t-il de ces processus pluriels de légitimation?

\section{COMPARER L'ACTION ORGANISÉE EN MATIÈRE D'INTERNATIONALISATION DE L'ENSEIGNEMENT SUPÉRIEUR: REMARQUES LIMINAIRES}

Avant de présenter les grammaires déployées en France et au Québec pour rendre compatibles des intérêts hétérogènes tout en répondant à des enjeux locaux, trois remarques liminaires s'imposent.

La première remarque liminaire est d'ordre sémantique et invite à un éclaircissement de notre posture théorique et méthodologique. Le secteur de l'enseignement supérieur est défini ici comme une configuration d'interdépendances entre des acteurs qui agissent à des niveaux d'action multiples (Musselin, 2001). La pluralité et l'hétérogénéité potentielle ainsi conférées à l'enseignement supérieur permettent d'éviter de focaliser trop l'attention sur un seul niveau d'action, par exemple les universités, et ainsi d'omettre les autres parties prenantes de l'action publique en matière d'enseignement supérieur: les ministères et les grandes organisations mondiales comme l'OCDE, les étudiants et leur famille, les entreprises et les organisations civiles, etc. ${ }^{12}$. Elles nous inscrivent dans une sociologie de

12. Si les analyses présentées ici peuvent sembler étato-centrées (elles n'engagent pas le vécu et les représentations d'acteurs autres qu'étatiques, objet de la deuxième phase de ce projet de recherche portant sur les parcours d'étudiants proprement dits), elles prennent toutefois acte de la multiplicité des acteurs et de leur niveau d'action au sein même de l'appareil d'État (voir la note méthodologique). Ce sont bien les écarts d'adhésion à l'internationalisation, les tensions entre les intérêts en jeu et les arrangements entre acteurs qui font l'objet de l'analyse. 
l'action organisée, où l'organisation fait référence "aux processus par lesquels une situation d'interactions se stabilise entre des acteurs individuels et collectifs, et donc, aux normes et arrangements que ces derniers ont développés (plus ou moins dans la durée) et à partir desquels ils produisent et entretiennent des modes de fonctionnement collectifs » (Musselin, 2005: 53).

A contrario des approches en matière d'analyse des politiques publiques, qui tendent à surévaluer l'importance des moments décisionnels et donc de la puissance publique, cela dans une perspective de «solution de problème " linéaire et descendante, l'approche en matière de sociologie de l'action organisée privilégiée ici s'intéresse à la variété des cadres cognitifs et des arrangements construits par les acteurs sociaux (Lascoumes et Le Galès, 2004; Michaud et Thoenig, 2009; Muller, 2000; Musselin, 2005). L'idée n'est pas de nier qu'il puisse y avoir un cadre régulateur commun à l'ensemble des acteurs de l'enseignement supérieur, mais plutôt de ne pas surestimer son caractère contraignant et déterminant en s'intéressant à l'hétérogénéité des représentations et des comportements à l'intérieur de ces limites.

\section{Note méthodologique sur les entretiens auprès des acteurs institutionnels}

Nous avons rencontré des représentants agissant à des niveaux différents de l'action publique, soient aux niveaux de l'administration des établissements, des organismes publics et des ministères. Au Québec, les représentants des universités œuvraient à l'Université du Québec à Montréal (UQAM), à HEC Montréal, à l'Université de Montréal et à l'Université Laval. En France, les établissements de l'enseignement supérieur représentés sont l'Université Vincennes-Saint-Denis (Paris 8), l'Université Paris-Diderot (Paris 7), l'Université de Versailles-Saint-Quentin-en-Yvelines (VSQY) et la Chambre de commerce et d'industrie de Paris (CCIP), laquelle chapeaute 10 écoles dans le champ du commerce et du management. Ces choix d'établissements universitaires tiennent à la fois de contraintes pratiques liées aux lieux d'installation des chercheures, du nombre important d'étudiants maghrébins qui y sont accueillis et d'une volonté de diversifier les champs de formation.

Au niveau des organismes publics québécois, nous avons rencontré des représentants de la Conférence des recteurs et des principaux des 
universités du Québec (CREPUQ) et de l'Agence universitaire de la francophonie (AUF). En France, ont été interrogées des personnes travaillant également à l'AUF, ainsi qu'à l'Agence CampusFrance, à l'Office méditerranéen de la jeunesse $(\mathrm{OMJ})$ et à la Conférence des présidents d'université (CPU). Enfin, au niveau ministériel nous avons rencontré des acteurs œuvrant, pour le volet québécois de la recherche, dans différents services du ministère de l'Éducation, du Loisir et du Sport (MELS), du ministère de l'Immigration et des communautés culturelles du Québec (MICC), du ministère des Relations internationales du Québec (MRI), du ministère Affaires étrangères, Commerce et Développement Canada ainsi que du ministère Citoyenneté et Immigration Canada (CIC). Pour le volet français, nous avons rencontré du personnel du ministère des Affaires étrangères et européennes (MAEE), du ministère de l'Enseignement supérieur et de la Recherche (MESR), ainsi que du ministère de l'Éducation nationale, de la Jeunesse et de la Vie associative (MENJVA). Les organismes, ministères et services ont été retenus en fonction du rôle qu'ils jouent en matière d'orientation des flux et d'accueil d'étudiants étrangers.

Les analyses ont été conduites de manière inductive (Deslauriers, 1997). Nous avons d'abord fait une "lecture flottante » des entretiens retranscrits, laquelle nous a permis de dégager de premières hypothèses (adhésion aux discours dominants en dépit de deux ordres de tension) (Mayer et Deslauriers, 2000). Nous avons ensuite construit une grille d'analyse recelant les principaux thèmes à explorer plus en profondeur, en vertu de ces hypothèses, lors d'une seconde lecture. Pour assurer l'anonymat des personnes rencontrées, et à la demande de plusieurs d'entre elles, tous les extraits d'entretien présentés dans cet article seront identifiés à partir d'un nom fictif, du niveau d'action (université, organisme ou ministère) et de la société représentée (France, Québec ou Canada).

Cela dit, et il s'agit de notre deuxième remarque liminaire, si nous nous intéressons aux fabrications symboliques entourant les processus d'internationalisation et de managérialisation de l'enseignement supérieur (dans sa version concrète qu'est l'accueil d'étudiants étrangers d'origine maghrébine), et si donc nous concevons ces processus d'abord comme des «idées" ou des « croyances", nous ne postulons pas qu'elles engendrent systématiquement les pratiques — même conçues comme multiples et hétérogènes. Rappelons ici qu'avec la « sociologisation » de 
l'approche des politiques publiques depuis les années 1980 (Doray et Maroy, 2008; Muller, 2000), les courants cognitifs d'analyse des politiques publiques se sont grandement développés. Ces derniers insistent sur le fait qu'une politique publique n'est pas seulement un programme technique, mais implique des « référentiels ( (Jobert et Muller, 1987), des " cognitions » (Michaud et Thoenig, 2009) qui orientent la définition des problèmes sociaux et des solutions. Ces approches, si elles ont l'avantage de sortir l'analyse des politiques publiques d'un cadre séquentiel et techniciste, ont parfois pour effet pervers de laisser à penser que l'univers des représentations induit des pratiques, en un lien de causalité unidirectionnel ${ }^{13}$. Or il peut se passer du temps avant que les idées puissent avoir des effets observables, si bien que le rapport de causalité est difficile à établir, élément sur lequel des travaux en sciences politiques insistent depuis longtemps (Meyer et Rowan, 1977). Plus fondamentalement encore, ce type d'approche conduit à la fiction que les idées et les pratiques seraient des éléments distincts qui n'ont pas à être analysés dans le même mouvement (Oger et Ollivier-Yaniv, 2003).

Dans cet article, nous nous attachons aux discours de légitimation complexes, parfois contradictoires, avancés par des acteurs situés pour expliquer leur participation à la mouvance de l'internationalisation des universités, cela en lien avec les dispositifs existant dans leur contexte. Notre perspective s'attarde donc aux idées, sans exclure les pratiques évoquées par les acteurs. Toutefois, nous ne préjugeons pas de l'engendrement initial de l'un par l'autre. Les schèmes cognitifs sur l'internationalisation de l'enseignement supérieur peuvent influer sur les pratiques tout comme il est possible que les pratiques invitent les acteurs institutionnels rencontrés à développer une rhétorique argumentative à leur sujet (Musselin, 2000). L'objectif est donc moins de faire une sociogenèse de la manière dont une idée (ou un dispositif) s'impose, et encore moins de statuer de sa véracité ou de son efficacité. L'intérêt est de mettre au jour, dans une visée comparative, les configurations de logiques discursives potentiellement différenciées à l'œuvre à un moment et dans deux sociétés donnés pour justifier de la participation, par le prisme de l'accueil d'étudiants maghrébins, à l'internationalisation de l'enseignement supérieur dans une perspective de plus en plus marchande.

Ce volet comparatif de la recherche fait l'objet de notre troisième remarque liminaire. Comment dégager des singularités nationales sans,

13. Pour un aperçu des débats sur la relation entre idées et pratiques dans le champ de l'action publique, voir Musselin (2000). 
d'une part, généraliser outrancièrement les effets de connaissance produits (d'autant lorsque l'enquête est microsociologique) et sans, d'autre part, culturaliser les différences? L'approche sociétale privilégiée offre des pistes afin de contourner ces pièges (Maurice, Sellier et Silvestre, 1982). Contre une perspective fonctionnaliste qui postule une continuité de tendances lourdes et déterminantes dans une très grande majorité de sociétés, et contre une approche culturaliste qui cherche au contraire à ériger des modèles nationaux distincts à tendance essentialisante, l'approche sociétale s'intéresse aux interactions et aux jeux d'influence réciproque entre de multiples acteurs et institutions sociales en vue de dégager des "cohérences » nationales (Lallement, 2003). Ces cohérences sociétales ont émergé de nos analyses lorsque des modalités semblables de légitimation traversaient le discours d'une pluralité d'acteurs différenciés d'une même société et semblaient se distinguer des discours observés dans l'autre contexte sociétal.

Cela dit, pour ne pas présumer de différences entre le Québec et la France avant confrontation à l'empirie, nous n'avons pas systématiquement renvoyé dos-à-dos les deux sociétés et avons été attentives à des proximités transversales, notamment selon les catégories d'acteurs et les niveaux d'action en jeu. Du reste, étant donnée la focale microscopique empruntée, il est certainement plus judicieux de comprendre cette enquête comme une étude exploratoire dont les configurations de discours de légitimation sont susceptibles d'être interprétées en termes sociétaux (Vassy, 2003).

\section{TENSIONS ET LÉGITIMATIONS COMPLEXES DU «MARCHÉ » INTERNATIONALISÉ DE L'ENSEIGNEMENT SUPÉRIEUR}

Deux ordres de tension liée à l'internationalisation de l'enseignement supérieur, plus spécifiquement au recrutement et à l'accueil des étudiants internationaux, émergent tant en France qu'au Québec du discours des acteurs institutionnels rencontrés. Ils donnent chacun à voir des modalités complexes de légitimation de la participation à cette mouvance globale et laissent entrevoir des traitements différenciés de la mobilité des étudiants marocains, algériens et tunisiens.

\section{Logique marchande contre vocation culturelle des universités}

Dans la logique marchande et concurrentielle qui prévaut depuis quelques années au Québec comme en France dans le secteur de l'enseignement 
supérieur, le recrutement des étudiants internationaux est majeur pour des raisons économiques et de positionnement culturel et scientifique sur la scène internationale. Cette logique entre toutefois en tension avec la mission culturelle et linguistique des universités, cela en France autant qu'au Québec.

\section{La langue de formation: obstacles et stratégies}

Dans les deux sociétés et quel que soit le niveau d'action des acteurs rencontrés, on admet que la langue française, si elle favorise le recrutement d'étudiants tunisiens, algériens et marocains, est plutôt un frein au recrutement d'étudiants internationaux en provenance du Brésil, de Russie, d'Inde et de Chine (les BRIC), considérés pourtant comme vecteurs indispensables d'une internationalisation toujours plus poussée et souhaitable:

Parce qu'il paraît intéressant d'avoir accès, justement, pour le coup... une logique un peu de marché... enfin, le marché global de l'enseignement, c'est dit, faut être présent sur ces nouveaux terrains. Parce qu'on sait, la Chine est déjà devenue l'un des premiers pays en termes de nombre d'étudiants et ça augmente encore...Les grands flux se situent là. Donc on peut estimer que ça a un impact sur le développement des universités, des projets de recherche, donc il faut être prêt. (Laurent Lerouge, Ministère, France)

Toutefois, cette perception de la langue française de formation comme obstacle au recrutement d'étudiants internationaux n'a pas le même degré d'imprégnation des discours en France et au Québec et fait l'objet d'appropriations et de stratégies de contournement différenciées.

Au Québec, le maintien d'une offre de formation en français est assumé comme un choix politique et certains regrettent d'ailleurs qu' « internationalisation » rime trop souvent, dans l'esprit de leurs vis-àvis à l'étranger, avec anglicisation :

Par exemple, aux pays scandinaves [...], ils disent « on s'est internationalisés, on donne des cours en anglais ». Nous on pense que c'est un peu...il faut que ce soit, euh... tu sais, que ce soit un peu plus nuancé que ça. Dans ce sens-là. Mais il y a une tendance à ce que l'internationalisation soit synonyme d'anglais, puis on n'est pas d'accord avec cette position-là. (Donald Lalonde, Université, Québec) 
Dans les universités comme dans les ministères et organismes publics, les acteurs rencontrés au Québec ont développé un discours de valorisation du français en lui adjoignant un caractère anglo-saxon lié au système universitaire québécois ainsi qu'à la proximité de l'anglais, dans le but d'en faire un élément de "distinction » sur le "marché » international de l'enseignement supérieur. C'est d'ailleurs en ces termes que de nombreuses institutions se présentent sur Internet et dans les documents officiels: "caractère francophone ", "positionnement résolument américain " (Université de Montréal); " première université francophone d'Amérique " (Université Laval); "Le Québec, c'est l'espace francophone au cour des Amériques ", "Carrefour des cultures latines et anglo-saxonnes » (MELS).

Dans cet effort pour réinvestir de sens la langue française comme langue attractive de formation, la référence à l'Europe, et souvent explicitement à la France, «notre compétiteur » (Michel Desrochers, Université, Québec), est récurrente :

De plus en plus, les pays du Maghreb choisissent le Québec et le Canada parce qu'ils veulent sortir du modèle français. Donc ils choisissent un modèle américain, mais qui parle français. C'est au Québec. (Philippe Savage, Ministère, Québec)

Nous verrons dans la prochaine sous-section que l'importance de la langue française revendiquée par les acteurs québécois peut tout à fait aller de pair avec la défense de l'anglicisation des programmes par les mêmes acteurs comme stratégie de recrutement intensif dans les BRIC. Les contradictions apparentes de l'internationalisation de l'enseignement supérieur à tendance marchande s'observent donc aussi au sein d'un même acteur et témoignent de la flexibilité du cadre d'interprétation et d'action : c'est parce qu'il défend l'un des éléments de la mouvance d'ensemble (ex. : réaffirmer l'importance du fait français dans l'accueil d'étudiants étrangers) que ce dernier peut adhérer par ailleurs à certains autres (ex. : recruter des étudiants anglophones des BRIC).

En France, le jalon comparatif n'est pas la francophonie. Lorsqu'on mesure son positionnement dans l'espace international de l'enseignement supérieur, ce sont les pays anglo-saxons, à commencer par les États-Unis, qui constituent le référent ultime : «c'était un public qui nous échappait un peu au détriment des États-Unis, au détriment de l'Angleterre » (Bernard Muhr, Organisme, France). Ici, la perception qu'ont les acteurs institutionnels du français comme langue de formation et de son rôle dans le recrutement d'étudiants internationaux est plutôt associée aux liens 
historiques qui unissent la France à ses anciennes colonies, incluant les pays du Maghreb. La francophonie apparait surtout comme un "devoir» envers «l'ancien empire colonial " puisque "l'histoire nous a investis de responsabilités durables et auxquelles on ne peut en aucun cas renoncer » (Julien Pastel, Ministère, France). Dans ce dernier cas, la langue française et les relations historiques avec les anciennes colonies ne sont pas sans apparaître comme des atouts "malgré elles ", voire une contrainte dans un contexte où on cherche à diversifier la provenance des flux d'étudiants internationaux, notamment des pays émergents : «[...] on cherche à rééquilibrer un peu les flux, je dirais, traditionnels, historiques, de mobilité » (Louis Breuillard, Ministère, France).

\section{Entre les bassins « traditionnels » et les « marchés prioritaires », la valse du recrutement}

Dans les deux sociétés, on peut noter des positions discursives et des pratiques ambiguës face au recrutement à l'international, qui sont à mettre en relation avec les rapports respectifs du Québec et de la France à la francophonie internationale et avec leurs propres politiques d'immigration.

Au Québec, on reconnaît que le Maghreb constitue un "marché naturel » (Gisèle Goulet, Organisme, Québec) d'étudiants étrangers, et que le recrutement y est «relativement facile, honnêtement » (David Fortin, Université, Québec). La région est une sorte de bassin acquis qu'on ne veut pas perdre pour des raisons linguistiques et d'immigration de travail, en conformité avec les intentions du $\mathrm{MICC}^{14}$. Sur la scène fédérale, il semblerait, selon ce représentant, que les interlocuteurs québécois aient été peu enclins, initialement, à collaborer avec le Gouvernement canadien dans un champ de compétence qui ne lui est pas réservé par la constitution.

On a communiqué avec le gouvernement du Québec. On leur a demandé : «dites-nous c'est quoi vos marchés prioritaires ». Ils ont toujours refusé de nous les donner. [...] Moi je me rappelle, il y a une dame qui s'appelait [Mme X], qui était la [personne responsable], qui était archi souverainiste, et qui ne le cachait pas,

14. Dès 1991, le ministère affirmait sa volonté d'assurer la pérennité du français à travers sa politique en matière d'immigration et d'intégration (MCCIQ, 1991). Plus récemment, il a assuré vouloir encourager l'installation au Québec des étudiants étrangers, arguant que ces derniers «ont eu le temps de s'y acclimater et [que] leurs diplômes sont reconnus beaucoup plus facilement par les employeurs québécois » (MICC, 2008: 18). 
et qui disait ouvertement que pour elle, le gouvernement fédéral avait pas d'affaire là (Jocelyn Castonguay, Ministère, Canada).

Si le Maghreb fait partie de la liste des régions prioritaires d'ÉduCanada pour la promotion de son enseignement supérieur, ce serait dû à l'initiative personnelle du fonctionnaire chargé de la question à Ottawa et de son souci de maintenir des relations cordiales avec Québec:

Pour moi, le problème c'était que l'Égypte, je savais pas comment mettre ça. C'était pas assez important pour mettre en tant que pays prioritaire, mais j'avais comme un petit... Parce que j'avais été placé en Égypte, je savais que c'était un bon marché, je voulais les aider... Mais je pouvais pas justifier de mettre l'Égypte comme marché prioritaire. Alors ce que j'ai fait, c'est que j'ai dit ÉgypteMaghreb, on le met ensemble : Afrique du Nord. [...] Alors c'est comme ça que le Maghreb est devenu un marché prioritaire (Jocelyn Castonguay, Ministère, Canada).

En France, on met de l'avant qu'en maintenant des liens de coopération universitaire avec le Maroc, l'Algérie et la Tunisie, on restreint l'exode des cerveaux, voire l'immigration. Au paradigme de l'exode des cerveaux, on souhaite opposer celui de leur circulation, à l'instar du dispositif APS présenté plus haut, qui encourage en définitive le retour dans le pays d'origine. En offrant un appui aux réformes de l'université des pays du Maghreb, ces derniers pourront entrer « dans le concert européen d'enseignement supérieur» (Daniel Clermontel, Organisme, France). Certains estiment que si les flux d'étudiants en provenance du Maghreb ont baissé, c'est parce que « les structures de formation supérieure locales se sont, dans ces dernières années, très fortement développées » (Louis Breuillard, Ministère, France). Honorer son devoir envers les anciennes colonies permettrait en dernière analyse de freiner la venue des étudiants étrangers en France.

Les relations solides qui unissent le Maghreb au Québec et à la France font en sorte qu'on consacre plutôt les efforts de recrutement aux pays émergents. Pour ce faire, on a recours dans les deux sociétés à une diversité de stratégies. Comme première stratégie, on cible des zones prioritaires de recrutement: "On n'exclut pas des nationalités, mais on exclut des zones... En espérant le plus possible les zones stratégiques les plus intéressantes : Europe, Amérique du Nord et les BRIC... Brésil, Russie... » (JeanLouis Grosjean, Université, France). Une deuxième stratégie consiste à modifier le calendrier de recrutement : des acteurs rencontrés en France nous ont dit avoir avancé les dates annuelles de recrutement dans certaines 
zones non francophones où ils perdaient les candidats au profit des universités anglo-saxonnes. Cette procédure n'est probablement pas sans effet sur les étudiants des zones dites francophones, à commencer par le Maghreb, qui sont soumis au test de classement français TAGE MAGE (plutôt qu'à son versant anglophone GMAT), qui a lieu plus tard dans l'année. Comme une première vague de sélection a déjà été faite dans les régions où est majoritairement pratiqué le GMAT, il reste moins de places pour les étudiants ayant effectué le TAGE MAGE. Les étudiants du Maghreb ne sont donc pas favorisés par ce mode de recrutement : " Eux ils passent le TAGE MAGE, mais le concours est encore plus sélectif pour eux puisque maintenant on favorise le GMAT, donc... » (Jean-Louis Grosjean, Université, France).

Une autre stratégie consiste à signer des accords avec les pays visés. Certaines universités mettent également en place une structure permettant l'offre de cours de français dans le pays d'origine, particulièrement en Chine, avant le départ. Un dernier moyen est de multiplier les cours en anglais dans les programmes. Cette dernière mesure est particulièrement présente dans les disciplines liées à la gestion et à l'administration, où des programmes sont dispensés en anglais, à la fois en France et au Québec. Dans ces derniers cas, les admissions de candidats non francophones pour suivre un programme en anglais se trouvent facilitées par l'offre de cours de français pendant les études. L' "impératif pour avoir le diplôme " est alors d' " avoir un niveau de maîtrise de la langue française » suffisant non pas au début, mais bien au terme des études (Didier Bastien, Université, France).

Certaines tensions sont toutefois repérables autour de l'anglicisation des programmes. En France, où la langue française d'enseignement est moins conçue qu'au Québec comme un choix politique, certains se sentent limités dans leurs actions d'internationalisation par la Loi relative à l'emploi de la langue française (loi Toubon) adoptée en 1994, laquelle crée notamment l'obligation de rédaction en langue française. D'autres réduisent les difficultés à angliciser davantage les cursus aux lacunes du corps professoral en anglais: "Les Français sont peu polyglottes ... je pense que ça tient surtout à ça » (Thierry Bertrand, Université, France). Au Québec, des actions de résistance ont été menées par des étudiants locaux quand des universités ont eu des velléités d'angliciser certains programmes :

Ils avaient lancé une politique linguistique qui disait en substance: "nos étudiants ne doivent pas uniquement prendre des langues étrangères, ils doivent faire des cours dans leur discipline en langue étrangère et on va permettre l'emploi d'autres 
langues »... [...] Ils ont été obligés de se retirer, ça a été un échec cuisant. [...] La personne qui veut lancer des projets et les associations étudiantes montent au créneau assez rapidement. (David Fortin, Université, Québec)

En somme, le Maghreb est conçu comme une zone de recrutement naturel pour le Québec et la France. Pour les deux sociétés, toutefois, les zones prioritaires de recrutement, c'est-à-dire celles où l'on consacre la majeure partie des ressources, sont incontestablement les pays émergents tels que la Chine, l'Inde et le Brésil, futures puissances technologiques, scientifiques et économiques jugées incontournables.

\section{Vers une instrumentalisation de l'étudiant international?}

La soumission de l'internationalisation des universités à des objectifs commerciaux s'observe non seulement à travers les ambivalences vis-à-vis de la langue de formation et le prisme des régions priorisées, mais aussi des filières de formation et des niveaux d'études privilégiés.

Au Québec, à tous les niveaux d'action on dit accorder la prévalence aux étudiants des $2^{\mathrm{e}}$ et $3^{\mathrm{e}}$ cycles et des chercheurs postdoctoraux, des niveaux jugés «à forte valeur ajoutée » (Marc Dumas, Université, Québec). "Ce qui a été décidé, c'est de réduire au minimum les exemptions [des droits de scolarités supplémentaires] affectées au premier cycle pour [les] mettre davantage sur les $2^{\mathrm{e}}$ et $3^{\mathrm{e}}$ cycles » (Philippe Savage, Ministère, Québec).

Trois principales justifications sont avancées pour expliquer cette prérogative allouée aux étudiants des cycles supérieurs. La première concerne la nécessité, pour le maintien de certaines filières de formation et d'emploi délaissées par les étudiants québécois, comme l'informatique, de recruter des étudiants à l'étranger. La seconde a trait à la volonté de développer la recherche dans les universités québécoises ainsi que des liens de coopération entre les universités québécoises et les universités étrangères. Enfin, la troisième justification s'inscrit plus concrètement dans une logique économique. En privilégiant les cycles supérieurs, les diplômés issus de l'étranger sont mieux à même de contribuer à l'essor de l'économie nationale et sont plus « rentables » pour l'État:

Il y a une étude d'Immigration Canada, il y a quelques années, qui a vu que si on compare un immigrant qui arrive comme ça de l'étranger ou un étudiant qui a gradué d'une université et qui décide de rester après ses études, cinq ans après qu'il devient 
résident permanent, l'étudiant qui a eu des études au Canada fait 10 ooo \$ de plus de salaire que l'immigrant qui a fait ses études à l'étranger. Ça, ça démontre qu'il a eu une plus grande facilité à entrer dans le marché du travail et qu'il a eu plus de succès à essayer de gravir les échelons. Ça veut dire que c'est un meilleur immigrant. Donc dans ce contexte-là, c'est sûr qu'avoir des étudiants qui décident de rester, c'est mieux pour la société canadienne $[\ldots]$ parce que les coûts de société pour l'intégration sont beaucoup moindres. (Jocelyn Castonguay, Ministère, Canada)

Parce qu' « ils se font des amis, ils ont des relations, ils connaissent le Québec, ils ont une expérience québécoise, de la culture québécoise », on suppose que les étudiants étrangers s'intégreront plus facilement comme immigrants. "Alors il y a un programme [Programme de l'expérience québécoise] qui est mis sur pied pour ces étudiants-là, pour qu'ils puissent faire une demande et ils vont passer prioritairement $»^{15}$ (Caroline Portelance, Ministère, Québec). "En une semaine, deux semaines, je pense, ils ont leur CSQ [Certificat de sélection du Québec] » (Roland Meunier, Ministère, Québec). Cette situation génère un certain mécontentement chez les acteurs universitaires rencontrés, puisque leur invisibilité statistique ne permet pas de montrer l'ampleur réelle de l'internationalisation du campus, ces étudiants ne venant pas « ajoute[r] à l'expérience» (Michel Desrochers, Université, Québec).

Cela dit, il est également souhaité au Québec qu'une autre partie des diplômés retournent dans leur société d'origine afin qu'ils contribuent au rayonnement de l'enseignement supérieur québécois, à l'établissement de réseaux internationaux de recherche scientifique et au développement de relations diplomatiques et commerciales avec le Québec.

En France, la primauté accordée aux candidats étrangers des cycles supérieurs est également le fruit d'une décision politique : «il y a un objectif clairement assumé qui est d'attirer plutôt des étudiants de niveau master, doctorat. Réaffirmé dans tous les discours de notre ministre " (Laurent Lerouge, Ministère, France). De même qu'au Québec, on souhaite développer la vocation de recherche de l'université et on juge que

15. Le Programme de l'expérience québécoise permet aux travailleurs et diplômés qui satisfont à certaines exigences, ainsi qu'à leur conjoint et enfants à charge, d'obtenir en accéléré un certificat de sélection du Québec, soit le document officiel d'immigration délivré par le gouvernement du Québec. Ce document est un préalable à leur admission par le gouvernement du Canada, en vertu du partage des responsabilités Québec-Canada (MICC, 2007). 
les diplômés des niveaux master et doctorat sont plus susceptibles de contribuer à l'attractivité de l'enseignement supérieur français, lorsqu'ils retournent dans leur pays d'origine, que ceux de licence. Certains s'appuient d'ailleurs concrètement sur leur réseau d'anciens diplômés pour effectuer leurs activités de recrutement :

Nous on s'appuie beaucoup là-dessus sachant que c'est surtout la communauté HEC qui nous aide parce qu'elle est la plus nombreuse, parce que c'est là où il $\mathrm{y}$ a le sentiment d'appartenance, de communauté et de représentation la plus importante et la plus ancienne et qu'ils sont un peu partout dans le monde. (Jean-Louis Grosjean, Université, France)

À la différence de la situation au Québec, toutefois, les acteurs institutionnels français présentent trois autres ordres d'argumentation pour légitimer la priorité accordée aux cycles supérieurs. Premièrement, on avance des motifs d'imputabilité vis-à-vis de l'opinion publique française :

Enfin, pour parler très clairement, voire crûment, [...] c'est le contribuable français qui assume les frais de scolarité de la totalité des étudiants étrangers que nous accueillons. Donc s'ils veulent faire des choix budgétaires, si je puis dire, dans ce qu'ont à assumer les contribuables, on préfère le niveau master-doctorat. On considère que ça participe plus au rayonnement de l'université. Il y a plus de perspectives, effectivement, de coopération. (Laurent Lerouge, Ministère, France)

Deuxièmement, l'intérêt pour les étudiants des niveaux master et doctorat s'accompagne davantage en France qu'au Québec d'une rhétorique de l'« excellence» :

[P] ourquoi faire de la promotion [au Maghreb] puisque de toute façon tous les matins j'ai 1000 étudiants devant ma porte? [...] Oui, effectivement, il y a peut-être 1 ooo étudiants, mais peut-être que les plus intéressants sont déjà... voilà, déjà partis, ou vont partir aux États-Unis, en Angleterre, au Canada. Donc voilà. C’est ceux-là qu'on veut aussi, bien évidemment comme tout le monde, en priorité, et pas forcément avoir que des étudiants qui ont eu des refus de la part d'autres pays et qui "se rabattent" sur la France, entre guillemets. (Bernard Muhr, Organisme, France)

La course aux meilleurs étudiants, dans un contexte de compétition mondiale, nécessite des dispositifs d' "écrémage » (François Durand, Organisme, France) et de sélectivité accrue. Ici, la procédure CEF, sur laquelle 
nous reviendrons dans la section suivante, est considérée comme jouant ce rôle de triage : «Les CEF, d'une certaine façon, c'est un petit peu comme une garantie » (Natalie Rivail, Université, France).

Enfin, le troisième ordre de justification qui diffère de celle entendue auprès des acteurs institutionnels rencontrés au Québec concerne l'immigration. Contrairement au Québec, où l'immigration pour études et l'immigration de travail sont de plus en plus articulées au niveau politique, en France « ça n'a jamais été écrit tel quel dans la politique » (Laurent Lerouge, Ministère, France). A contrario, des stratégies sont mises en place au niveau des organismes publics et des ministères pour favoriser en amont le retour des diplômés dans leur pays d'origine : «On aura tendance à privilégier des formations qui nous semblent, et les pays émetteurs aussi, naturellement plus utiles pour le pays d'origine, en particulier lorsqu'il s'agit d'attribuer des bourses " (Julien Pastel, Ministère, France). On souhaite également favoriser les "mobilités encadrées " (Laurent Lerouge, Ministère, France), davantage jugées garantes d'un retour en vertu du maintien des liens institutionnels qu'elles favorisent avec la société d'origine.

Il est intéressant de comparer l'accent que mettent les représentants des instances publiques que nous avons rencontrés au Québec et en France sur le recrutement aux cycles supérieurs avec les flux réels d'étudiants internationaux. Au niveau du premier cycle, le nombre d'étudiants internationaux a crû de $16,1 \%$ au Québec contre seulement 6,8 \% en France. À la maîtrise, l'évolution est pour la même période de $11,4 \%$ au Québec et de $8,2 \%$ en France. C'est au doctorat que l'écart entre les deux sociétés est le plus grand: on observe en effet un accroissement de $41,4 \%$ des étudiants internationaux inscrits au doctorat au Québec, mais de seulement 2,6 \% en France. Les visées consistant à accroître le nombre d'étudiants aux cycles supérieurs sembleraient davantage se réaliser dans la province canadienne qu'en France.

Ces tendances concernant l'accueil d'étudiants internationaux au niveau du doctorat au Québec et en France sont encore plus accentuées lorsqu'on considère la région émettrice du Maghreb. Tandis que le nombre de doctorants maghrébins augmente de 55,5\% au Québec entre 2007 et 2010, il diminue de 9,6 \% en France (tableaux 3 et 4$)^{16}$.

16. À titre indicatif, les étudiants maghrébins inscrits au doctorat augmentent de façon similaire dans les universités anglophones québécoises, avec un taux de $40 \%$ (MELS, 2011). 
TABLEAU 3 Évolution des effectifs d'étudiants maghrébins dans les universités québécoises francophones, par niveau, de 2007 à 2010

\begin{tabular}{|l|c|c|c|c|c|c|c|c|c|}
\hline & \multicolumn{2}{|c|}{$\mathbf{2 0 0 7 - 2 0 0 8}$} & \multicolumn{2}{|c|}{$\mathbf{2 0 0 8 - 2 0 0 9}$} & \multicolumn{2}{|c|}{$\mathbf{2 0 0 9 - 2 0 1 0}$} & \multicolumn{2}{|c|}{ 2010-2011* $^{*}$} & Évolution \\
\hline & $\mathbf{N}$ & $\mathbf{\%}$ & $\mathbf{N}$ & $\mathbf{\%}$ & $\mathbf{N}$ & $\mathbf{\%}$ & $\mathbf{N}$ & $\%$ & \\
\hline $\mathbf{1}^{\text {er }}$ cycle & 1025 & 60,8 & 1072 & 58,3 & 1103 & 54,4 & 1012 & 53,4 & $-1,3 \%$ \\
\hline $\mathbf{2}^{\mathbf{e}}$ cycle & 541 & 32,1 & 648 & 35,2 & 762 & 37,6 & 697 & 36,8 & $28,8 \%$ \\
\hline $\mathbf{3}^{\text {e }}$ cycle & 119 & 7,1 & 119 & 6,5 & 163 & 8,0 & 185 & 9,8 & $55,5 \%$ \\
\hline Total & 1685 & 100 & 1839 & 100 & 2028 & 100 & 1894 & 100 & $12,4 \%$ \\
\hline
\end{tabular}

Source: MELS (2011)

* Données provisoires

TABLEAU 4 Évolution des effectifs d'étudiants maghrébins dans les universités françaises, par niveau, de 2007 à 2010

\begin{tabular}{|l|c|c|c|c|c|c|c|c|c|}
\hline & \multicolumn{2}{|c|}{$\mathbf{2 0 0 7 - 2 0 0 8}$} & \multicolumn{2}{|c|}{$\mathbf{2 0 0 8 - 2 0 0 9}$} & \multicolumn{2}{|c|}{$\mathbf{2 0 0 9 - 2 0 1 0}$} & \multicolumn{2}{|c|}{ 2010-2011* } & Évolution \\
\hline & $\mathbf{N}$ & $\mathbf{\%}$ & $\mathbf{N}$ & $\mathbf{\%}$ & $\mathbf{N}$ & $\mathbf{\%}$ & $\mathbf{N}$ & $\%$ & \\
\hline $\mathbf{1}^{\text {er }}$ cycle & 18655 & 36,7 & 18774 & 37,0 & 19973 & 37,6 & 19861 & 37,4 & $6,5 \%$ \\
\hline $\mathbf{2}^{\mathrm{e}}$ cycle & 25987 & 51,0 & 25875 & 51,1 & 27190 & 51,2 & 27564 & 52,0 & $6,1 \%$ \\
\hline $\mathbf{3}^{\mathrm{e}}$ cycle & 6239 & 12,3 & 6019 & 11,9 & 5903 & 11,1 & 5638 & 10,6 & $-9,6 \%$ \\
\hline Total & 50881 & 100 & 50668 & 100 & 53066 & 100 & 53063 & 100 & $4,3 \%$ \\
\hline
\end{tabular}

Source: Agence CampusFrance, fiches sur l'Algérie (2011a), le Maroc (2011b) et la Tunisie (2011c)

Notons que pour cette même période, l'effectif des étudiants chinois au doctorat s'accroît de 161,7 \% au Québec et de 42,2 \% en France ${ }^{17}$ (Agence CampusFrance, 2011d: 1). Les nombres absolus n'ont certes pas le même ordre de grandeur au Québec qu'en France, ce qui se répercute sur les taux d'évolution. Ces quelques données peuvent toutefois nous informer sur les tendances qui se dessinent actuellement. L'accroissement des étudiants internationaux au troisième cycle semble grandement bénéficier aux

17. Par comparaison, les taux d'évolution des étudiants chinois aux autres cycles sont les suivants : 11,9 \% au Québec et 30,4 \% en France pour le premier cycle; $-14,9 \%$ au Québec et -5,9 \% en France pour le deuxième cycle. Dans les universités anglophones québécoises, leur croissance est modeste au $1^{\text {er }}$ cycle $(4,6 \%)$ comme au $2^{\mathrm{e}}$ cycle $(3,6 \%)$, mais remarquable au $3^{\mathrm{e}}$ cycle $(87,7 \%)$. 
étudiants chinois, tant en France qu'au Québec. Elle paraît également profiter plutôt bien aux étudiants du Maghreb pour le cas du Québec. En revanche, ces derniers ne font pas partie des contingents qui viennent grossir les rangs des doctorants en France. Si ces évolutions contrastées entre les cycles d'études et les nationalités des étudiants sont à relier aux visées et aux incitatifs mis en place dans les sociétés de départ, elles font également écho aux orientations manifestées par les acteurs que nous avons rencontrés tant en France qu'au Québec.

Cela étant dit, les idéaux économiques qui sous-tendent l'internationalisation de l'enseignement supérieur font parfois l'objet de stratégies de contournement ou d'abstention de la part des acteurs du monde universitaire. Par exemple, certains opèrent une disjonction entre internationalisation et marchandisation et parviennent à trouver des partenaires qui ne soient pas entièrement voués à une logique commerciale tout en répondant aux besoins des étudiants :

[C]'est très difficile d'avoir des coopérations $[\ldots]$ avec des universités anglophones dans des pays anglophones, parce qu'aux ÉtatsUnis c'est marchand, en Angleterre c'est marchand [...]. Donc on a cherché ailleurs des partenariats avec des universités, par exemple aux Pays-Bas, où il y a une bonne formation en anglais. (Natalie Rivail, Université, France)

D'autres refusent de se soumettre à la prescription qui consiste à privilégier les cycles supérieurs au nom de la philosophie de leur institution : «nous on est tentés de penser que... c'est surtout lié au, à [notre] identité [...]. On doit recevoir tout le monde. Donc, quelqu'un veut venir dans notre université, il a droit de venir. [...] C'est vrai en licence [...] » (Thierry Bertrand, Université, France). Un représentant nous a confié qu'un service de son université acceptait sans grande condition de fournir des lettres d'invitation nominatives, jusqu'à ce que la préfecture s'inquiète du nombre de visas délivrés pour cette université.

Quoi qu'il en soit, les mesures de recrutement des étudiants à l'étranger s'accompagnent majoritairement, au Québec comme en France, d'un travail discursif autour de la volonté de resserrer la sélection des étudiants internationaux. Elles tendent parallèlement à déclasser certains d'entrée, selon leur pays d'origine, leur niveau d'études, la filière envisagée et l'inscription ou non dans un dispositif institutionnel de mobilité. On est moins dans une logique d'attractivité "à tout prix » que dans une logique élective, ce dont semble témoigner, notamment, l'évolution croissante des flux d'étudiants étrangers au niveau du doctorat au Québec. 


\section{Injonction à l'accroissement des flux contre surveillance accrue des étudiants internationaux}

Les prescriptions qui semblent prévaloir dans le secteur universitaire autour de l'internationalisation de l'enseignement supérieur, à commencer par l'accroissement de l'accueil d'étudiants étrangers, s'accompagnent d'un impératif de gestion du risque migratoire. Tant en France qu'au Québec, les acteurs institutionnels rencontrés semblent s'accorder sur la nécessité d'identifier et de sanctionner le «faux étudiant». En revanche, les deux sociétés ont recours à des discours de légitimation ainsi qu'à des stratégies différenciées pour donner un sens à ce second ordre de tension.

Selon une majorité d'acteurs institutionnels français, l'État met de l'avant la sélection en amont des étudiants étrangers comme un moyen privilégié de prévenir l'entrée de faux étudiants.

Nous avions des déperditions importantes à l'entrée en France de jeunes gens qui venaient en France avec un pseudo projet d'études universitaires, et que vous ne voyez après plus jamais dans un cadre académique universitaire. Ils disparaissaient dans la nature complètement. Pas besoin de vous dire que tout ceci présentait des inconvénients tout à fait évidents. (Julien Pastel, Ministère, France)

Pour l'État français, les études ne sont pas considérées comme un moyen d'immigrer. Le potentiel de retour dans le pays d'origine est contrôlé dès en amont, via la procédure CEF. Cette procédure implique d'abord la constitution d'un dossier électronique personnel, moyennant des frais de dossiers (1 ooo Dirhams, soit environ 115 \$ CAN, pour le Maroc). Plusieurs protagonistes interviennent tout au long de la préparation du dossier de candidature. Dans l'ordre : 1) les espaces CampusFrance, en soumettant le candidat à un test de langue, en faisant l'examen administratif du dossier (dont la vérification de l'authenticité des diplômes) et en organisant un entretien sur le "projet personnel» de l'étudiant ${ }^{18}$; 2) le

18. À titre indicatif, une simulation d'inscription sur le site de CampusFrance nous a permis de voir qu'un candidat originaire du Maroc doit fournir : un bordereau de paiement des frais CampusFrance, sa Carte d'Identité Nationale, son diplôme de baccalauréat, les relevés de ses notes les années de baccalauréat, les relevés de ses notes pour les études subséquentes, ses diplômes d'enseignement supérieur obtenus, sa convocation au concours ou à un entretien en France (le cas échéant), une attestation de stage (le cas échéant), le tout en français et en copie certifiée conforme. Le candidat doit joindre également un $\mathrm{CV}$, un projet d'études, un projet professionnel ainsi qu'un énoncé de motivation. 
Service de coopération et d'action culturelle de l'Ambassade (SCAC), qui donne un avis en ligne sur le projet d'études; 3) l'établissement d'enseignement supérieur visé, qui sélectionne (ou non) le candidat et lui accorde, selon le cas, une pré-inscription en ligne et enfin 4) le service consulaire, qui décide de l'attribution ou non du visa.

Certains représentants de tous les niveaux insistent sur le fait que la procédure CEF ne vise pas à limiter les flux d'étudiants étrangers en provenance de pays spécifiques: "alors pour régler tout de suite un malentendu, le système des espaces à procédure CEF, ce n'est pas du tout une façon d'introduire des quotas qui concerneraient je ne sais quel type de population. Absolument pas. Il n'y a aucune espèce de quota " (Julien Pastel, Ministère, France). En privilégiant les étudiants qui présentent un projet d'étude en adéquation avec leur cursus antérieur, "l'enjeu de la procédure CEF est de favoriser les parcours de réussite en France " (Adèle Berjolet, Ministère, France). Les agents des CEF semblent jouer un rôle d' "orienteur " (Samira Alphonse, Université, France), qui sera consolidé, si on se fie aux propos de cet interlocuteur, par des formations officielles :

Et donc on est en train de travailler sur le programme, mais $a$ priori ça serait sur vraiment former les agents CampusFrance à l'orientation. Comment mener un entretien d'information ou d'orientation à l'étudiant. Comment percevoir ses envies, parce que c'est vrai que c'est par là ...l'étudiant sait pas toujours ce qu'il veut faire pendant toute sa vie. Voilà, pour ça on fait appel à des experts. (Bernard Muhr, Organisme, France)

$\mathrm{Si}$, au départ, les établissements d'enseignement supérieur français étaient réticents à s'engager dans la procédure CEF pour des raisons techniques et politiques - « est-ce que les rôles étaient suffisamment bien délimités entre l'ambassade, l'université... " (Samira Alphonse, Université, France) —, aujourd'hui elles y verraient un avantage sur le plan administratif.

L'expérience a montré que la dématérialisation des dossiers des candidats étrangers était un gain de temps considérable, que le contrôle documentaire effectué en amont était plus fiable que celui [que les établissements] pouvaient effectuer depuis la France et que les commissions pédagogiques avaient accès à tous les dossiers, quel que soit leur niveau, leur permettant ainsi d'exercer pleinement leur pouvoir de décision en matière de pré-inscription. (Adèle Berjolet, Ministère, France) 
Ici, l'opérateur CampusFrance semble avoir été un acteur majeur dans la production de l'idée que le marché supérieur de l'enseignement supérieur était devenu une évidence et une nécessité :

Donc vraiment la problématique à l'époque c'était : un, il faut assurer la meilleure promotion possible de nos études supérieures, première chose, et deuxième chose, adopter une attitude un petit peu plus commerciale vis-à-vis des prestations à fournir d'abord aux établissements d'enseignement supérieur et éventuellement auprès des étudiants. On était dans une dynamique assez nouvelle en France que le directeur de l'époque peut-être, le directeur général de l'époque d'ÉduFrance, n'a pas bien su pousser. Ce qui fait qu'on a eu besoin de changer et de directeur général, et d'institution pour réussir mieux dans cette entreprise. C'est comme ça que CampusFrance est né. (Julien Pastel, Ministère, France)

On le voit par cet extrait, la rhétorique de l'enseignement supérieur comme marché international a exigé, pour exister et s'imposer dans un pays comme la France, un "médium » (CampusFrance), lui-même porté par des acteurs qui voyaient un intérêt à le faire vivre.

Certains acteurs ministériels admettent néanmoins que la procédure CEF représente un moyen global de réduire la venue de "faux étudiants » dans les pays considérés les plus à risque, dont font partie les pays du Maghreb qui lui sont assujettis :

On ne peut pas faire une enquête de contre-espionnage pour chaque candidat à l'immigration [...]. Alors on se réfugie en disant pas... pas après, pas avant la licence ou un master, pas dans les disciplines qui ne sont pas prioritaires, pas dans les universités qui n'ont pas déjà des accords avec les universités du pays... On essaye de rationaliser notre peur et/ou notre manque de connaissance. (Stéphane Kraemer, Ministère, France)

De plus, d'après l'étude ethnographique de Spire (2009) réalisée dans l'un des trois pays du Maghreb qui nous intéressent, le pouvoir dévolu en amont aux « conseillères » embauchées pour faire l'étude des dossiers ainsi que les multiples échelons à travers lesquels voyage la décision, qui tendent à déresponsabiliser tout un chacun, feraient en sorte que les commissions disciplinaires des universités n'auraient plus vraiment leur rôle pédagogique à jouer dans l'évaluation des qualifications de l'étudiant. En outre, les ressources financières de l'étudiant sont un critère additionnel de contrôle, ce que viennent confirmer les propos de cet autre interlocuteur : 
"L'idée, c'est aussi d'attirer des étudiants solvables, donc qui paient et qui viennent de pays plus en développement ou de pays émergents et qui donc vont apporter, vont quelque part aussi enrichir la France » (François Durand, Organisme, France) ${ }^{19}$.

Aussi, certains acteurs universitaires s'inquiètent de la diminution du taux d'acceptation des étudiants étrangers soumis à la procédure CEF :

On leur demande par exemple, il me semble, l'attestation de naissance, là... un certificat de naissance. Pour ceux d'entre eux qui viennent de très, très loin, c'est pas évident de le trouver. Et du coup, finalement, c'est un ...c'est une sélection à l'argent, c'est certainement la première chose et puis une sélection à la débrouille, une sélection à ....aux gens qui ont la plus grande facilité, finalement. Ceux pour lesquels c'est facile, ce sera facile. Mais je vous dit ça... mais je sais qu'en Algérie, donc, il y a énormément de refus. (Thierry Bertrand, Université, France)

D'autres soulignent que la procédure CEF, parce qu'elle est majoritairement assignée à des pays à forte pression migratoire et ayant généralement des systèmes éducatifs moins performants, est considérée comme un label disqualifiant. La procédure CEF a provoqué des protestations diplomatiques dans certains pays, notamment au Canada qui depuis a été soustrait de la liste des pays à procédure CEF. Enfin, certains acteurs universitaires soulignent les injonctions contradictoires auxquelles ils doivent parfois faire face :

C'est peut-être ça qui est plus gênant dans... dans nos rapports avec le MAEE, parce que je pense que le MAEE, avec le Ministère et CampusFrance, veut promouvoir les universités françaises, mais qu'en même temps on limite le nombre de gens qui peuvent venir en France. (Natalie Rivail, Université, France)

À titre d'exemple, alors que les étudiants iraniens sont « des ingénieurs qu'on s'arrache à prix d'or ", ces étudiants voient leur demande de visa presque systématiquement refusée : "Nous nous bagarrons tous les jours avec le "militaire" que nous avons ici et qui oppose son veto à toutes les demandes de visa des étudiants iraniens » (Stéphane Kraemer, Ministère, France).

19. La solvabilité paraît faire l'objet d'un contrôle moindre au Québec dans la mesure où les étudiants qui y viennent effectivement, « c'est pas nécessairement la classe qui court après leurs [sic] sous noirs » (Gisèle Goulet, Organisme, Québec). 
Il règne donc autour de la procédure CEF certaines ambiguïtés qui traversent l'ensemble des niveaux d'action. Cette procédure est perçue à la fois comme une mesure de contrôle des flux et comme un dispositif facilitant l'accueil des étudiants. Elle est perçue tantôt comme un service d'accompagnement des étudiants vers la réussite, tantôt comme un vecteur d'inégalités d'accès à l'enseignement supérieur français. Enfin, elle contrevient aux efforts de recrutement auxquels les acteurs sont par ailleurs sommés de se soumettre.

À l'instar des discours de légitimation identifiés pour le contexte français, les acteurs institutionnels québécois et canadiens aspirent aussi à une meilleure surveillance des flux d'étudiants sur le territoire : «Il semble que les niveaux soient très variables et que les étudiants viennent ici parce qu'ils ont de l'argent. Ils viennent de familles qui ont de l'argent et ils ont obtenu de très très bonnes notes dans leurs écoles, mais on sait pas s'ils ont payé ou si c'est des notes... parce que ça aussi, ça s'arrange » (David Fortin, Université, Québec).

Pour cela, le gouvernement fédéral travaille actuellement à la mise sur pied d'un programme visant à effectuer un suivi des étudiants sur le territoire canadien afin de s'assurer de l'intégrité du programme de permis d'études. "So we actually don't know whether students are studying, whether they are attending classes or if they are doing another kind of activity. [...] We try to ensure that those students that come here are genuine» (Gale Racine, Ministère, Canada). Cette attention du fédéral portée à l'immigration déguisée est toutefois reçue avec prudence en contexte québécois, et ce pour trois raisons principales. Premièrement, à l'instar des discours tenus en France, les acteurs universitaires et ministériels québécois, y compris d'ailleurs certains du gouvernement fédéral, notent une contradiction importante entre les diverses actions d'Ottawa. D'un côté, le gouvernement fédéral cherche à augmenter le nombre d'étudiants étrangers en organisant d'importants salons de recrutement: "Our view is that we view international students as, you know, as one of the more ideal immigrants " (Gale Racine, Ministère, Canada). L'offensive en matière de promotion de l'éducation canadienne à l'étranger, avec Édu-Canada et l'élaboration d'une image de marque, en témoignent. De l'autre côté, le ministère de l'Immigration attribue au compte-gouttes des permis d'études aux candidats en provenance de certains pays : "It is seen that there is a bit of a disconnect between marketing initiatives and CIC in the way that we allow entry for international students " (Gale Racine, Ministère, Canada). « [E]ffectivement, on se demande pourquoi eux [le Gouvernement fédéral] organisent des missions pour qu'on aille recruter là-bas alors qu'ils ne 
soutiennent... qu'il y a pas d'ouverture pour accepter ces étudiants-là » (Danielle Audet, Université, Québec). Les stratégies de recrutement des universités sont par conséquent affectées par la manière dont CIC distribue les visas étudiants : " avant d'aller recruter dans des pays où ils sont à $10 \%$ de taux d'obtention, on va aller à ceux qui sont à $95 \%$. Parce que ça veut dire que sur 10 personnes à qui je vais faire une offre, il y en a juste une qui va avoir son permis » (Gisèle Goulet, Organisme, Québec).

Les acteurs québécois sont également réticents à s'associer aux actions promotionnelles du gouvernement canadien parce que l'éducation est une compétence provinciale et qu'ils jugent avoir acquis une longue expertise leur permettant de recruter avec succès leurs propres étudiants internationaux. D'après ce représentant rencontré à Ottawa, la légitimité du fédéral dans le secteur de l'enseignement supérieur n'était pas acquise non plus au sein même du gouvernement canadien. La naissance d'Edu-Canada tient davantage à la contingence et aux compromis politiques qu'à un processus instrumental destiné, tel que le veut la conception des approches « classiques » des politiques publiques, à mettre en ouvre un projet explicitement défini en amont par des décideurs pour résoudre le "problème » de l'attractivité de l'enseignement supérieur canadien à l'étranger :

Paul Martin avait fait un deal avec le NPD pour rester au pouvoir un certain temps sur un budget pis le NPD avait dit qu'il fallait remettre je sais pas combien de millions ou milliards à l'éducation. Mais ils avaient pas dit pour quoi en éducation. Alors ils ont mis sur pied un groupe de travail interministériel pour dire : ok, on travaille en éducation [...]. Donc quand le gouvernement sait pas quoi faire, il crée un comité. Alors ils ont créé un comité pis j'étais sur le comité. Et moi je leur ai dit : ben, une place où est-ce qu'on a une responsabilité, c'est en éducation internationale parce qu'on est responsable des relations internationales du Canada. Alors j'ai dit : peut-être qu'on pourrait travailler là-dedans. Ils ont dit : excellente idée, au moins on a la juridiction. (Jocelyn Castonguay, Ministère, Canada)

Sans ministère de l'Éducation, le gouvernement fédéral a donc inscrit les activités promotionnelles de l'éducation dans le secteur du commerce international. Or, même les délégués commerciaux étaient peu affables à l'idée de faire la promotion de l'éducation et il a fallu l'insistance de la personne responsable du service :

Il y avait des délégués commerciaux qui étaient des vieux de la vieille. Pour eux, l'éducation, c'est pas un secteur économique, 
donc ils lui donnaient pas beaucoup d'importance. Mais elle, elle a dit: " non non non, un instant! C'est très important l'éducation, pis oui vous le mettez ». (Jocelyn Castonguay, Ministère, Canada)

Aujourd'hui, le rapprochement opéré par le gouvernement fédéral entre éducation et commerce ne fait pas de doute si l'on en veut pour preuve le registre entrepreneurial sur lequel Édu-Canada présente ses services ${ }^{20}$.

Néanmoins, les acteurs de l'enseignement supérieur québécois commenceraient à « respecter " Edu-Canada parce que cela leur donne une visibilité profitable : «ça leur coûte rien, c'est moi qui paye pour tout » (Jocelyn Castonguay, Ministère, Canada). Certains admettent en effet s'accommoder de l'ingérence du fédéral dans un domaine de prédilection provinciale et s'engagent dans ses initiatives, notamment les salons, en développant des stratégies pour en tirer avantage :

quand tu fais un salon avec le Canada, tu dis pas la même chose aux personnes qui sont devant toi que quand c'est un salon qui est organisé par le Québec, parce que quand tu fais un salon qui est organisé par Édu-Canada, tu leur dis : « il faut que vous prouviez, quand vous demandez votre permis d'études, que vous allez retourner chez vous ». (Danielle Audet, Université, Québec)

Dans les deux sociétés, et à tous les niveaux d'action, les acteurs institutionnels qui n'ont pas "la sécurité nationale dans [leur] mandat" (Danielle Audet, Université, Québec) se sentent contraints dans leur recrutement par les politiques étatiques de contrôle migratoire. La situation est probablement d'autant plus contradictoire pour l'État québécois que ce dernier cherche depuis quelques années une meilleure articulation entre ses politiques d'immigration et ses politiques d'accueil d'étudiants étrangers. Or, en raison de la répartition des compétences entre le fédéral et les provinces au sein de la constitution canadienne, les efforts au Québec sont parfois contrecarrés par Immigration Canada.

20. «Édu-Canada PRO fournit des renseignements en ligne sur le secteur de l'éducation aux clients du domaine par l'entremise du Délégué commercial virtuel du MAECI. Une fois que vous vous serez connecté et que vous aurez choisi vos marchés, vous pourrez accéder à des études de marché, des renseignements sur les activités, des statistiques et des nouvelles régionales ", citation extraite du site du Gouvernement du Canada sur le Service des délégués commerciaux, version juin 2012 (c'est nous qui soulignons). 


\section{CONCLUSION}

Les cadres d'interprétation de l'internationalisation de l'enseignement supérieur et de son caractère marchand, bien qu'ils soient le fruit d'une construction sociale, tendent à s'autonomiser et à s'imposer aux acteurs si l'on en juge la faible remise en question dont ils font l'objet par les personnes que nous avons rencontrées. Cela n'empêche toutefois pas les divers acteurs institutionnels de se trouver au centre de contradictions et de tensions. Au Québec comme en France, nous avons observé que la mission culturelle et linguistique de l'université entrait en tension avec la logique de marché qui lui sert aujourd'hui de substrat. Des deux côtés de l'Atlantique, le français comme langue de formation et comme source d'identité collective, de même que la mission d'aide au développement des universités, se heurtent à des impératifs de recrutement dans les «BRIC » et la recherche de l'excellence à des fins stratégiques et économiques. Autant au Québec qu'en France, l'ouverture à l'international va ainsi de pair avec une surveillance accrue des flux d'étudiants étrangers.

D’un point de vue transversal aux deux sociétés d'accueil ici étudiées, ces idées divergentes sont parfois portées par un même individu, et souvent par des acteurs provenant d'organisations différenciées. Les personnes et institutions qui ont pour fonction de participer à la promotion de l'internationalisation de l'enseignement supérieur, notamment, se trouvent parfois en porte à faux avec celles qui sont en charge de l'immigration. Nous avons toutefois vu que certains acteurs faisaient montre de stratégies, conformément à l'esprit de leur université et à leurs convictions personnelles, afin de contourner certaines contraintes.

D'un point de vue comparatif, si l'idée de marché internationalisé de l'enseignement supérieur apparaît comme quelque chose d'inéluctable, comme une forme de détermination au demeurant produite bien au-delà des États, il n'empêche que les sociétés peuvent emprunter des chemins différenciés pour y adhérer et y répondre. Aussi les procédés de légitimation sont-ils le fruit de logiques de production situées socialement. Tandis qu'au Québec le français est un choix politique et fait partie d'une réappropriation dans le discours pour en faire un trait distinctif promotionnel par rapport à d'autres destinations francophones, en France la fidélité à la francophonie apparaît davantage comme un devoir et une contrainte dans un monde dominé par l'anglais. Tandis qu'au Québec l'impératif est de recruter des étudiants des cycles supérieurs pour qu'ils contribuent à la recherche et au développement économique des universités et de la société québécoises, en France la grammaire gravite surtout autour de l'excellence. 
Tandis qu'au Québec l'étudiant étranger est vu soit comme le parfait immigrant, soit comme celui avec qui on pourra faire des affaires à son retour dans son pays d'origine, en France l'étudiant étranger est censé retourner dans son pays une fois diplômé afin de contribuer au rayonnement de la culture française. Les acteurs développent ainsi des sous-univers de sens flexibles leur permettant d'adhérer à l'accueil d'étudiants étrangers - à commencer par les étudiants d'origine maghrébine - sur fond de compétitivité marchande, selon la mission qui leur est dévolue et le contexte sociétal. C'est par le biais de ces réappropriations, en fonction des interactions sociales et des intérêts en jeu, que l'idée de l'internationalisation de l'enseignement supérieur dans une perspective marchande arrive à prendre forme et à s'imposer localement.

Ces logiques discursives identifiées au niveau sociétal, en appui avec certains instruments d'action politique, concourent à l'émergence de catégories normatives, c'est-à-dire de symboles, touchant le marché internationalisé de l'enseignement supérieur. Au Québec, il tend à se dessiner la catégorie du "bon immigrant ", à savoir l'étudiant étranger diplômé d'un cycle supérieur dans un établissement universitaire québécois qui s'intègre facilement sur le marché du travail de la société d'accueil, et donc à moindre coût pour l'État. En France, les modalités d'adhésion au marché internationalisé de l'enseignement tendent plutôt à voir se profiler la catégorie du «bon étudiant étranger " : l'étudiant de cycle supérieur, dont le dossier scolaire est parmi les meilleurs, qui vient parfaire sa formation dans un établissement d'enseignement supérieur français pour ensuite retourner faire profiter son pays d'origine de ses qualifications, cela en contribuant au rayonnement culturel et scientifique ainsi qu'à l'essor politique et économique de la France sur la scène internationale. Concrètement, soit au vu des tendances migratoires de ces dernières années dans les deux sociétés, les étudiants en provenance du Maghreb semblent mieux se fondre dans la catégorie québécoise du bon immigrant que dans celle du bon étudiant étranger en France. Des recherches ultérieures intégrant les discours et les dispositifs sur la mobilité étudiante sortante dans les sociétés de départ permettraient de circonscrire de manière plus juste la part véritablement dévolue aux dispositifs de migration et d'accueil en France et au Québec dans l'évolution des flux observée ces dernières années dans ces deux sociétés, de même qu'elles pourraient dévoiler, dans une perspective ouverte par l'histoire croisée (Werner et Zimmermann, 2003), les phénomènes d'interdépendance et de réversibilité entre États de départ et États d'arrivée. 
L'étude microscopique des processus de légitimation d'acteurs étatiques aux positions différenciées, parce qu'elle fait montre d'un ensemble d'arrangements pour adhérer à la rhétorique qu'est le marché internationalisé de l'enseignement supérieur, doit-elle nous laisser conclure que cette rhétorique, et celle sur la mobilité internationale des étudiants maghrébins, est "sans adversaire ", c'est-à-dire sans groupes d'opposants (Juhem, 2001) ? Il apparaît que non, au regard de trois éléments observés au cours de cette recherche et qui mériteraient d'être creusés plus finement dans d'autres enquêtes, notamment ethnographiques. Le premier concerne les conduites individuelles de distanciation par rapport aux activités prescrites, lesquelles font montre de stratégies d'opposition, notamment à la visée marchande de l'internationalisation et à ses effets de déclassement. Le deuxième a trait à la dichotomie fédéralprovincial du côté canadien, qui met en scène un jeu redistributif des ressources et des pouvoirs susceptibles d'appeler à des confrontations politiques. Enfin, avec l'exemple des réactions à l'anglicisation des cursus, les étudiants et les professeurs apparaissent sans aucun doute comme les acteurs clés d'une résistance aux visées néolibérales (Winter et O'Donohue, 2012) qui sous-tendent certaines des activités d'internationalisation de l'enseignement supérieur. La perspective microscopique empruntée ici nous a permis de constater l'élasticité des rhétoriques actuelles sur l'internationalisation et la managérialisation de l'enseignement supérieur, qui facilite les arrangements et leur traduction effective dans des contextes socio-historiques différenciés. En revanche, elle offre également des prises pour la critique sociale en dévoilant leurs modulations situées (contre une vision de nécessité), ainsi que les tensions susceptibles d'être exploitées pour d'éventuelles actions de résistance.

\section{BIBLIOGRAPHIE}

Agence CampusFrance. 2011a. "Afrique \& Maghreb : Algérie », Fiche Pays CampusFrance 2011. Paris, Agence CampusFrance.

Agence CampusFrance. 2011b. "Afrique \& Maghreb: Maroc», Fiche Pays CampusFrance 2011. Paris, Agence CampusFrance.

Agence Campusfrance. 2011c. "Afrique \& Maghreb: Tunisie, Fiche Pays CampusFrance 2011. Paris, Agence CampusFrance.

Agence CampusFrance. 2011d. " Asie: Chine », Fiche Pays CampusFrance 2011. Paris, Agence CampusFrance.

Agence CampusFrance. 2011e. "Les étudiants internationaux », Chiffres clés CampusFrance 2011. Paris, Agence CampusFrance. 
Agence CampusFrance. 2011f. "La mobilité des étudiants du Maghreb, de l'Égypte et du Levant », Les notes CampusFrance, hors-série 2. Paris, Agence CampusFrance.

Aвdous, M. 2011. " Towards a framework for business process reengineering in higher education ", Journal of Higher Education Policy and Management, 33, 4: 427-433.

Agasisti, T. et G. Catalano. 2006. "Governance models of university systems - towards quasi-markets? Tendencies and perspectives: A European comparison ", Journal of Higher Education Policy and Management, 28, 3 : 245-262.

Borgogno, V. et J. Streiff-FénART. 1997. "L'accueil des étudiants étrangers en France : évolution des politiques et des représentations », Cahiers de l'URMIS, $2-3: 73-88$.

Bruno, I. 2008. À vos marques, prêts... cherchez! La stratégie européenne de Lisbonne, vers un marché de la recherche. Bellecombe-en-Bauges, Éditions du Croquant.

Castells, M. 2001. "La société en réseaux », dans L'ère de l'information, Paris, Fayard.

Conseil National pour le Développement de la Mobilité Internationale Des Étudiants (CNDMIE). 2004. Stratégies de développement de la mobilité internationale des étudiants et attractivité de l'enseignement supérieur français. Rapport pour l'année 2003-2004. Paris, CNDMIE.

Conseil Supérieur de l'Éducation (CSE). 2005. La mobilité internationale des étudiants au sein des universités québécoises. Sainte-Foy, CSE.

Coulon, A. et S. Paivandi. 2003. Les étudiants étrangers en France : l'état des savoirs. Paris, L'Observatoire national de la vie étudiante.

Deslauriers, J.-P. 1997. "L'induction analytique », dans J. Poupart (dir.), La recherche qualitative. Enjeux épistémologiques et méthodologiques, Montréal, Éditions Gaëtan Morin : 293-308.

Doray, P. et C. Maroy. 2008. "Présentation », Sociologie et sociétés, 40, 1 : 11-29.

Freitag, M. 1995. Le naufrage de l'Université. Et autres essais d'épistémologie politique. Québec, Nuit blanche / Paris, La Découverte.

GARnEAU, S. 2008. "Inégalités d'accès à l'espace international de l'enseignement supérieur et aux marchés du travail », dans S. MAzzella (dir.), L'enseignement supérieur dans la mondialisation libérale. Une comparaison internationale Maghreb, Afrique, Canada et France, Paris, Maisonneuve et Larose/IRMC: 133-150.

Giroux, A. 2006. Le pacte faustien de l'université. Montréal, Liber.

Glaser, B. et A. Strauss. 2012. La découverte de la théorie ancrée. Stratégies pour la recherche qualitative. Paris, Armand Colin.

Godin, B. 2006. «The Knowledge-based economy : conceptual framework or buzzword? », Journal of Technology Transfer, 31:17-30.

Gouvernement du QuéBec. 2002. Pour réussir l'internationalisation de l'éducation... Une stratégie mutuellement avantageuse. Québec, Gouvernement du Québec. 
Hassenteufel, P. 2005. «De la comparaison internationale à la comparaison transnationale. Les déplacements de la construction d'objets comparatifs en matière de politiques publiques ", Revue française de science politique, 55, 1 : 113-132.

Jobert, B. et P. Muller. 1987. L'État en action. Paris, Presses universitaires de France.

Juhem, P. 2001. «La légitimation de la cause humanitaire : un discours sans adversaires ", Mots, 65 : 9-27.

Lallement, M. 2003. "Pragmatique de la comparaison », dans M. Lallement et J. Spurk (dir.), Stratégies de la comparaison internationale, Paris, CNRS Éditions : 297-306.

Lascoumes, P. et P. Le Galès. 2004. "L'action publique saisie par les instruments ", dans P. Lascoumes et P. Le Galès (dir.), Gouverner par les instruments, Paris, Presses de Sciences Po : 11-44.

Legrand, L. 1988. Les politiques de l'éducation. Paris, Presses universitaires de France.

Maurice M., F. Sellier et J.-J. Silvestre. 1982. Politique d'éducation et organisation industrielle en France et en Allemagne : essai d'analyse sociétale. Paris, Presses universitaires de France.

M.A.U.S.S. 2009. L'Université en crise. Mort ou résurrection? Revue du MAUSS $n^{o}$ 33. Paris, La Découverte.

Mayer, R. et J.-P. Deslauriers. 200o. «Quelques éléments d'analyse qualitative : l'analyse de contenu, l'analyse ancrée, l'induction analytique et le récit de vie », dans R. Mayer, F. Ouellet, M.-C. St-Jacques et D. Turcotte (dir.), Méthodes de recherche en intervention sociale, Boucherville, Éditions Gaëtan Morin : 159-189.

Meyer, J. W. et B. RowAN. 1977. «Institutionalized organizations : formal structure as myth and ceremony", American Journal of Sociology, 83, $2: 340-363$.

Michaud, C. et J.-C. Thoenig. 2009. L'organisation et ses langages. Interpréter pour agir. Québec, Les Presses de l'Université Laval, 229 p.

Milot, P. 2003. "La reconfiguration des universités selon l'OCDE », Actes de la recherche en sciences sociales, 148, $1: 68-73$.

Ministère de L'Éducation, du Loisir et du Sport (MELS). 2011. "Portail informationnel, système GDEU [gestion des données sur l'effectif universitaire] », sur le site du MELS [données du 6 mai 2011]. www.mels.gouv.qc.ca

Ministère de l'Immigration et des Communautés culturelles (MICC). 2007. L'immigration au Québec. Partage des responsabilités Québec-Canada. Montréal, Direction des politiques, des programmes et de la promotion de l'immigration, Gouvernement du Québec.

Ministère de l'Immigration et des Communautés culturelles (MICC). 2008. Plan stratégique 2008-2012. Montréal, Direction des affaires publiques et des communications, Gouvernement du Québec. 
Ministère des Communautés culturelles et de l'Immigration du Québec (MCCIQ). 1991. Au Québec pour bâtir ensemble. Énoncé de politique en matière d'immigration et d'intégration. Montréal, Direction générale des politiques et programmes, Gouvernement du Québec.

Muller, P. 2000. "L'analyse cognitive des politiques publiques : vers une sociologie politique de l'action publique », Revue française de science politique, 50, 2 : 189-207.

Musselin, C. 2000. The role of ideas in the emergence of convergent higher education policies in Europe: the case of France. Cambridge (Mass.), Center for European Studies /Harvard University, Working Paper Series $\mathrm{n}^{\circ} 73$.

Musselin, C. 2001. La longue marche des universités françaises. Paris, Presses universitaires de France.

Musselin, C. 2005. "Sociologie de l'action organisée et analyse des politiques publiques : deux approches pour un même objet?", Revue française de science politique, 55, $1: 51-71$.

Musselin, C. 2008. "Vers un marché international de l'enseignement supérieur? », Critique internationale, 2, $39: 13-24$.

Oger, C. et C. Ollivier-Yaniv. 2003. "Analyse du discours institutionnel et sociologie compréhensive : vers une anthropologie des discours institutionnels ", Mots - Les langages du politique, 71 : 125-144.

Organisation de Coopération et de Développement économique (OCDE). 2004. Enseignement supérieur : internationalisation et commerce. Paris, Éditions OCDE.

Revel, J. 1996. " Micro-analyse et construction du social », dans J. Revel (dir.), Jeux d'échelles. La micro-analyse à l'expérience, Paris, Gallimard / Seuil : 15-36.

Simon, V. 2000. "La migration des étudiants maghrébins en France : une approche socio-historique (1962-1994)", dans V. GeIsser (dir.), Diplômés maghrébins d'ici et d'ailleurs. Trajectoires sociales et itinéraires migratoires, Paris, CNRS Éditions : 245-269.

Slama, S. 1999. La fin de l'étudiant étranger. Paris, L'Harmattan.

SPIRE, A. 2009. "Contrôler et choisir. La sélection des étudiants candidats à l'émigration vers la France », dans S. Mazzella (dir.), La Mondialisation étudiante. Le Maghreb entre Nord et Sud, Paris, IRMC/Karthala : 79-95.

VAssy, C. 2003. "Données qualitatives et comparaison internationale : l'exemple d'un travail de terrain dans des hôpitaux européens ", dans M. LALlement et J. SPURK (dir.), Stratégies de la comparaison internationale, Paris, CNRS Éditions : 215-227.

VinCENT-LANCRIN, S. 2008. «L'enseignement supérieur transnational : un nouvel enjeu stratégique?», Critique internationale, 2, 39:67-86.

Werner, M. et B. Zimmermann. 2003. «Penser l'histoire croisée : entre empirie et réflexivité », Annales. Histoire, sciences sociales, 58, $1:$ 7-36. 
Winter, R. P. et W. O’Donohue. 2012. "Academic identity tensions in the public university : which values really matter? ", Journal of Higher Education Policy and Management, 34, 6:565-573.

\begin{abstract}
Stéphanie GARNEAU and Caroline BOUCHARD

Complex legitimations in the internationalised higher education system: the mobility of students from the Maghreb to France and to Quebec

This article presents a comparative analysis from Quebec and France of the different modalities of agreement of the process of internationalisation of higher education. It deals specifically with one of the dimensions of this internationalisation, the mobility of foreign students, focusing on student flows which are significant in these two host societies: those from Morocco, Algeria and Tunisia. The presentation is based on interviews with thirty institutional actors, and on statistical data and public documents. The article shows that actors in France and Quebec are at the focal point of two major tensions: firstly between the logic of the market and the cultural and linguistic mission of universities; and secondly between the objective of increasing the numbers of foreign students and the need to manage the risks of immigration. Each of these tensions is shown to give rise to complex legitimation procedures in the attempt to reconcile them and to engage the different actors. The article sheds light on these legitimation processes through looking at the movements of students from the Maghreb to Quebec and France. Two normative categories - the "good immigrant" in Quebec and the "good foreign student" in France - are identified as tending to emerge as dominant as a result of these mental operations.
\end{abstract}

